\title{
Leitartikel
}

\section{Umweltethik und die Realität des Umwelthandelns am Beispiel Klimaschutz}

\author{
Werner Theobald \\ PD Dr. Werner Theobald, Ökologie-Zentrum der Universität Kiel, Olshausenstr. 40, D-24098 Kiel (wtheobald@ecology.uni-kiel.de)
}

DOI: http://dx.doi.org/10.1065/uwsf2004.06.082

\section{Zusammenfassung}

Ziel und Hintergrund: Der Beitrag behandelt die seit Jahren in den Umweltwissenschaften diskutierte Diskrepanz zwischen Einstellung und Verhalten. Er knüpft an die Diagnose an, dass diese Diskrepanz vor allem dadurch zu erklären sei, dass die Gewohnheiten des einzelnen, sein Lebensstil, seine Bequemlichkeit und sein Bedürfnis nach Wohlbefinden ihn (trotz vorhandenen Umweltbewusstseins) an einem umweltgerechten Verhalten hindern. Diese Diskrepanz wird moralphilosophisch reflektiert.

Schwerpunkte: Die Moralphilosophie behandelt seit der Antike die Frage, warum man trotz richtiger Ansichten gegen das als 'gut' und 'richtig' Erkannte verstößt. Die klassische Antwort auf diese Frage lautet: Wenn jemand wider Wissen gegen das 'Beste' handle, so sei er nicht im Besitze klaren Wissens, sondern er habe nur eine Meinung; um bloße Meinung und nicht klares Wissen handle es sich, wenn es nicht ein 'entschiedenes Urteil', sondern ein 'nachgiebiges' sei, das von der Lust überwältigt wird. Ethik verfolgt das Ziel, Verhaltensnormen durch den 'zwanglosen Zwang guter Argumente' zu begründen, sie zu fundieren; man könnte daher annehmen, dass sie zu 'entschiedenen Urteilen' führen kann. Diese Hypothese wird anhand ethischer Argumente für den Klimaschutz überprüft.

Ergebnisse und Schlussfolgerungen: Klimaschutzethik bzw. Umweltethik erweist sich bei näherer Betrachtung als strittiges Unternehmen, so dass von ihm die angenommene Sicherungsleistung nicht ausgehen kann. Letzteres resultiert auch daraus, dass die Umweltethik zu sehr auf die kognitive Überzeugungsleistung ihrer Argumente setzt; ethische Argumente müssen aber, damit sie handlungsleitend werden, auch emotionale Schichten ansprechen. Auf der Grundlage der Untersuchung naturpositiver Emotionen und Einstellungen wird diskutiert, wie die nötige 'Ansprache des Gefühls' erfolgen kann.

Schlagwörter: Aufklärung; Diskrepanz von Einstellung und Verhalten; Emotionen; ethische Argumente; Klimaschutz; Moralphilosophie; Politik; Umweltbewusstsein; Umwelthandeln; Vernunft

\section{Problemstellung}

Der amerikanische Psychologe L. Bickman führte vor gut 30 Jahren folgendes Experiment zum Umgang mit Abfall durch (Bickman 1972): Vor einer College-Bibliothek wurde mitten auf dem Fußweg eine zerknüllte Zeitung auf den Boden gelegt. Sie war so platziert, dass die Fußgänger darüber steigen oder um sie herum gehen mußten; es war also garantiert, dass die Vorbeikommenden auf den Abfall aufmerksam wurden. Ein Papierkorb stand in unmittelbarer Nähe. Jede fünfte Person, die daran vorbei ging, wurde zehn Meter weiter von einem Mitglied der Forschergruppe Bickmans angehalten. Man erklärte, dass man im Rahmen eines Ökologiekurses Interviews über das Abfallverhalten durchführe. Die entscheidende Frage, die den

\section{Abstract \\ Climate Protection. Environmental Ethics and the Reality of Environmental Behaviour}

This paper discusses the discrepancy of attitude and behaviour in an environmental protection from the point of view of philosophy. Coming from the classical thesis of moral philosophy, that someone who infringes the 'good' has no secure knowledge, it examines how ethical arguments can contribute to get such a knowledge. The result of this study is that the logical power of persuasion alone is not able to acquire such a knowledge; and in order to obtain practical efficiency, ethical arguments should converse to emotional stratifications of personality. Based on the examination of positive feelings for nature, the paper discusses how this can happen.

Keywords: Climate protection; discrepancy of attitude and behaviour; emotions; environmental awareness; environmental behaviour; environmental ethics; intellect; moral philosophy; politics; rationalism

Passanten im Verlauf der Interviews gestellt wurde, lautete: "Wenn Müll auf dem Boden liegt, sollte es dann in die Verantwortung aller fallen, den Müll aufzuheben, oder sollte dies die Angelegenheit der städtischen Straßenreinigung sein?" 94\% der Befragten antworteten, dass es in die Verantwortung aller fallen sollte, den Müll aufzuheben. Insgesamt wurden 506 Personen auf diese Weise befragt. Von ihnen hatten nur 8 Personen, d.h. ganze $1,4 \%$ tatsächlich den Müll aufgehoben.

Das Alltagsverständnis von Umweltbewusstsein spiegelt (ebenso wie die gängigen Vorstellungen der Umweiterziehung und Umweltpolitik) eine einfache Aufklärungsidee: Aus Wissen wird Einsicht und daraus das richtige Verhalten. Kurz: 'Vernunft wird siegen' (Haan/Kuckartz 1996, 103f.). Die sozialwissenschaftliche Umweltbewusstseinsforschung hat nun untersucht, wie stark diese angenommene 'Macht der Vernunft' im Bereich des Umweltschutzes wirklich ist. Sie förderte (ana$\log$ zu dem eben geschilderten Experiment) ein ernüchterndes Ergebnis zu Tage. Nahezu alle Studien, die in nationalen Kontexten wie im internationalen Vergleich durchgeführt wurden, zeigen, dass faktisch keine sonderlich hohe Korrelation zwischen dem Wissen, das man über Umweltprobleme hat, der daraus resultierenden Einsicht in nötige Verhaltensänderungen und dem Umweltverhalten selbst besteht. Die stärkste Beziehung besteht noch zwischen der sog. Handlungsbereitschaft und dem Umweltverhalten; d.h., wer die Absicht bekundet, sich durch seine Handlungen für die Umwelt einzusetzen, verhält sich auch umweltgerechter als jemand, der diese Absicht nicht äußert (obwohl man auch hier, wie das eingangs zitierte Beispiel zeigt, seine Zweifel haben kann). 


\section{Umweltbewusstsein in Deutschland}

Betrachten wir einige deutschlandspezifische Zahlen und Fakten. Die Bundesdeutschen rangieren, so international vergleichende Umfragen, in der Spitzengruppe der umweltbewussten Länder (Haan/Kuckartz 1996, 63). Was die Einschätzung der Frage angeht, ob Umweltprobleme ein sehr ernstes Problem im eigenen Land seien, belegen sie zusammen mit Suid-Korea sogar Platz 1 (Haan/Kuckartz 1996, 65). Repräsentative Untersuchungen zum Umweltbewusstsein in Deutschland sagen aus (Haan/Kuckartz 1996, 70):

- dass das Umweltbewusstsein in Deutschland kontinuierlich zugenommen hat,

- dass das Thema Umwelt zu den wichtigsten Themen der Zukunft gerechnet wird,

- dass die Bevölkerung zunehmend aufmerksam ist gegenüber Risiken und Gefährdungen, die durch die Umwelt verursacht werden und

- dass der Umweltschutz nicht nur als eine Aufgabe staatlicher Politik angesehen wird, sondern die überwiegende Mehrheit glaubt, selbst direkt etwas zur Verbesserung der Umwelt beitragen zu können.

Diesen durchweg positiven Einstellungen zum Thema Umweltschutz steht ein z.T. eklatant abweichendes Verhalten gegenüber, das oft bereits auf der Ebene der Verbalisierung der Handlungsbereitschaft anklingt. So stufen zwar nahezu $90 \%$ der bundesdeutschen Bevölkerung es als 'sehr wichtig' ein, die Umwelt vor Verschmutzung zu bewahren (Haan/Kuckartz 1996, 71) und knapp 70\% glauben, dass ein globaler Temperaturanstieg für sie 'sehr' bzw. 'extrem gefährlich' sei (Haan/ Kuckartz 1996, 69); aber nur weniger als $20 \%$ sind bereit, weniger Auto zu fahren (Haan/Kuckartz 1996, 81) - obwohl sie wissen, dass die durch das Autofahren entstehenden $\mathrm{CO}_{2}-$ Emissionen die Umwelt verschmutzen und den Treibhauseffekt wesentlich mit bewirken. Die Bereitschaft, weniger Auto zu fahren, rangiert in Deutschland sogar noch hinter der hypothetischen Bereitschaft, finanzielle Opfer für die Erhaltung des Regenwaldes zu erbringen (ebd.).

Nun ist es aber hauptsächlich der Verkehrssektor, der der Umweltpolitik Sorgen bereitet (SRU 2002, 223). Zwischen 1990 und 2000 war hier ein Anstieg der ohnehin schon beträchtlichen $\mathrm{CO}_{2}$-Emissionen um weitere $12,8 \%$ festzustellen, und nach neuesten Untersuchungen wird der $\mathrm{CO}_{2}$-Ausstoß des Straßenverkehrs in der Bundesrepubklik noch bis über das Jahr 2010 hinaus zunehmen (SRU 2002, 222). Selbst relativ niedrig aufgehängte Klimaschutzziele, die ohnehin in diesem Zeitraum als kaum erreichbar gelten, werden vor allem im Verkehrssektor deutlich verfehlt (SRU 2002, 223). Der Politik sind hier Grenzen gesetzt. Die ökologische Steuerreform, die kurzfristig (1999 bis 2001) einen Rückgang des Treibstoffabsatzes bewirkte und ein geschätztes Minderungspotential in Höhe von 6 bis 8 Mio. t $\mathrm{CO}_{2}$ generierte (ebd.), ist ständiger Kritik ausgesetzt ${ }^{1}$ und daher kaum weiter verschärfbar; ein Benzinpreis von 2-3 Euro pro Liter gar, der notwendig wäre, um die externen Kosten des Straßenverkehrs aufzufangen, schlichtweg nicht realisierbar - keine Regierung, gleich welcher Couleur, würde eine derartige Maßnahme überstehen.

Die am Beispiel des Verkehrsverhaltens besonders deutlich werdende Diskrepanz von Einstellung und Verhalten im Bereich des Umweltschutzes resultiert, wie man inzwischen weiß,

1 Wobei diese Kritik z.T. auch daher rührt, dass die finanziellen Einnahmen aus dieser Reform für sachfremde Leistungen (z.B. das Stopfen von Löchern der Rentenkasse) verwendet wurden. vor allem daraus, dass die Gewohnheiten des einzelnen, sein Lebensstil, seine Bequemlichkeit und sein Bedürnis nach spontanem Wohlbefinden ihn an einem umweltgerechten Verhalten hindern (Haan/Kuckartz 1996). ${ }^{2}$ Der Geist ist sozusagen willig, das Fleisch dagegen schwach. Nun wurde bereits in der antiken Moralphilosophie die Frage diskutiert, wie jemand wissentlich gegen das 'Beste handeln kann bzw. wie es möglich ist, dass man trotz richtiger Ansichten gegen das als 'gut' und 'richtig' Erkannte verstößt. Die klassische Antwort auf dieses sog. Akrasia-Problem stammt von Aristoteles und lautet: Wenn jemand wider Wissen "gegen das Beste handelt, so ist er nicht im Besitze klaren Wissens, sondern er hat nur eine Meinung." Um "bloße Meinung und nicht klares Wissen" handele es sich, wenn es nicht ein "entschiedenes Urteil", sondern ein "nachgiebiges" sei, das "von der Lust überwältigt wird" (Nikomachische Ethik VII 3, 1145b 34 - 1146a 1). Diese These enthält in Bezug auf unser Thema einiges an Wahrheit; denn es hat sich gezeigt, dass die sog. 'ökologisch Entschiedenen' in der Tat nach ihrer Einstellung handeln (Theobald 1998, 384) und dass bei ihnen die ansonsten verbreitete Tendenz nicht anzutreffen ist, die guten Vorsätze der 'Lust', dem Bedürfnis nach Bequemlichkeit und Wohlbefinden, zu opfern.

\section{Ethische Aspekte des Klimaschutzes}

Nun könnte man auf den Gedanken kommen, dass das philosophische Unternehmen einer Umweltethik, das seit Jahren unter Hochdruck betrieben wird, die bei den meisten Menschen anzutreffende Diskrepanz zwischen Einstellung und Verhalten im Bereich des Umweltschutzes überwinden helfen könnte. Erklärtes Ziel der Umweltethik ist es ja, Handlungsnormen zu begründen, sie zu fundieren, so dass man die Erwartung hegen könnte, dass auf diese Weise jene 'nachgiebigen' Einstellungen, von denen Aristoteles spricht, in 'feste', 'entschiedene' Einstellungen überführt werden könnten. Letzteres sollte, so könnte man meinen, möglich sein durch den 'zwanglosen Zwang guter Argumente', auf den die umweltethische Reflexion setzt. Schauen wir uns also ein einschlägiges Beispiel umweltethischer Reflexion einmal an. Ich beziehe mich wieder auf den Klimaschutz, da er zu den vorrangigen und dringlichen Aufgaben des Umweltschutzes gehört (SRU 2002, 210).

Klimaschutz, darüber sind sich die meisten Umweltethiker einig, impliziert Verpflichtungen gegenüber zukünftigen Generationen. Wie solche Verpflichtungen ethisch begründet werden können, ist jedoch in höchstem Maße strittig (Ott 2002 , 151ff.). Je nach Begründungsansatz bzw. ethischem Modell variieren die Argumente und Optionen, und es gibt sogar Ansätze, die - mit (ihrer Meinung nach) 'guten' Gründen bestreiten, dass wir überhaupt Verpflichtungen gegenüber zukünftigen Generationen hätten. Diese sog. 'No obligation'Argumente beruhen im wesentlichen auf folgenden Überlegungen (nach Ott 2002, 153 u. 157):

- Wir wissen nicht genau, wer zukünftige Menschen sein und was sie wollen werden; d.h. wir kennen ihre Bedürfnisse und Interessen nicht genau genug, um zu wissen, welche Hinterlassenschaften wir ihnen schuldig sind.

2 Hier muss man allerdings auch differenzieren; dort, wo es Infrastrukturprobleme gibt (z.B. im ländlichen Bereich, wo gut funktionierende Busoder Bahnanbindungen fehlen), kann man es sicher nicht der Bequemlichkeit der Menschen anlasten, wenn sie - etwa, um zur Arbeit zu kommen - das Auto benutzen. 
- Rechtsansprüche gründen aber in Bedürfnissen und Interessen. Wo diese nicht genau identifizierbar sind, gibt es keine konkreten Rechtsansprüche und damit auch keine konkreten Pflichten.

- Als ảbstrakte Pflicht gegenüber zukünftigen Personen könnte man zwar die Pflicht annehmen, zukünftige Personen zur Existenz zu bringen; wenn aber diese 'Hervorbringungspflicht' nicht geteilt wird - was wahrscheinlich ist, da sie absurd klingt -, entfallen niederrangige Pflichten wie beispielsweise die Hinterlassenschaft einer intakten Umwelt.

- Schließlich: Moralisch falsch handeln wir nur, wenn jemand Bestimmtes geschädigt wird. Das aber ist in Bezug auf zukünftige Generationen nicht der Fall. Es gibt somit keine 'Opfer', und wo es keine Opfer gibt, da gibt es keine Pflichten für ein bestimmtes moralisches Verhalten.

So streitbar diese Thesen und Argumente auch sein mögen, es bedarf z.T. erheblichen philosophischen Scharfsinns, sie zu entkräften (siehe Ott 2002). Dies aber koppelt zurück bzw. verstärkt den Eindruck, dass Umweltethik insgesamt ein höchst disparates Unternehmen ist - ein Unternehmen, das charakterisiert werden kann als das Ringen um die 'richtige' Ethik (im Bereich des Umweltschutzes). Da diese aber in höchstem Maße strittig scheint, kann von der Umweltethik die erhoffte 'Sicherungsleistung' offenbar nicht ausgehen; jene 'nachgiebigen' Einstellungen, von denen die Rede war, können durch sie nicht in 'feste', 'entschiedene' Einstellungen überführt werden.

\section{Zur Überzeugungskraft ethischer Argumente}

Ein zweites kommt hinzu: Moralische Argumente, sie mögen noch so gut theoretisch begründet sein, bewirken nicht per se Einstellungsänderungen von der Art, dass sie ein konsequentes Handeln nach sich zögen. Augustinus, einer der ersten Argumentationstheoretiker, hat dies in aller Klarheit erkannt. Er sagte: Damit 'sittliche' Argumente, also Argumente, die darauf aus sind, ein bestimmtes Verhalten zu bewirken, damit solche Argumente ihr Ziel auch erreichen, dürfen sie nicht nur rational überzeugen, sondern sie müssen ihre Adressaten auch 'rühren' (Augustinus 1925, 187). Mit anderen Worten: Moralische Argumente müssen, damit sie handlungsleitend werden, auch emotionale Schichten ansprechen. Nur bzw. erst Emotionen, das wissen selbst Pop-Stars wie Peter Maffay, öffnen die Menschen und machen sie empfänglich.

Nun hat die Moderne ein überaus zwiespältiges Verhältnis zum Gefühl. Einerseits, in ihren ethischen Fundamenten auf ihm beruhend, verdankt sie ihm (nahezu) alles. Ohne die Aufwallung des Protests, ohne die 'Parteinahme des Gefühls' (Jonas), würde es so etwas wie die Menschenrechte nicht geben. Es war das Gerechtigkeitsgefühl, das die Menschen 1789 für 'Freiheit, Gleichheit und Brüderlichkeit' auf die Barrikaden gehen ließ; keine noch so gut begründete moralphilosophische bzw. ethische Theorie hätte dies zu leisten vermocht. Andererseits ist die Moderne, entsprechend ihrem aufklärerischen Grundimpetus, extrem gefühlsskeptisch bzw. -aversiv eingestellt. Sie setzt, wie eingangs gesehen, auf die 'Macht der Vernunft'.

Diese Abwertung des Gefühls zeigt sich auch auf der Ebene ethischer Reflexion.

Emotionen wird nur in eingeschränktem Maße eine ethische Bedeutsamkeit zugestanden, insofern sie (analog zur Rolle von Daten im Rahmen empirischer Theorien) dazu dienen können, Moraltheorien zu falsifizieren - beispielsweise dann, wenn solche Theorien sog. 'kontraintuitive Konsequenzen' haben (siehe z.B. Bayertz 1988, 95; Krebs 1997, 349); in 'positivem
Verstande', um an eine Formulierung Kants anzuknüpfen, wird ihnen aber ethische Bedeutsamkeit weigehend aberkannt. Wer sich im Rahmen umweltethischer Überlegungen von Emotionen oder gar Intuitionen leiten lässt, gilt nicht selten als naiv, unreflektiert oder sentimental (Birnbacher 1998).

Nun kann man beobachten, dass es eine Reihe von Emotionen - genauer: Intuitionen - gibt, die im Zuge der modernen Umweltkrise aufkamen und die potentiell in der Lage wären, diese Krise abzuschwächen, stünden sie nicht unter dem genannten Verdikt. Es handelt sich dabei um Intuitionen, die der Natur einen 'Eigenwert' zubilligen, der dazu auffordert, schonender mit der Natur umzugehen. Die für die Moderne charakteristische Abwertung des Gefühls führt jedoch dazu, dass solche potentiell umweltschützerischen 'Instinkte' verdrängt, ja, oft noch nicht einmal bewusst werden. Dass es sie aber gibt, und zwar in hohem Maße, zeigt beispielsweise die Analyse von Bildern, die in den Medien (nicht selten in der Werbung, denn sie arbeitet ja mit dem Unbewussten) verwendet werden. Jeder kennt die berühmte Bier-Werbung, die vor einem 'Tatort' oder vor der Übertragung eines bedeutenden Sportereignisses über den Bildschirm flimmert und ein Stück heiler Natur zeigt, unterlegt mit den sphärischen Klängen von Musik, in der Gefühle wie Sehnsucht und Wehmut anklingen. Die Wirkung solcher Bilder besteht darin, dass sie ein atchaisches Verhältnis zur Natur reanimieren, in dem sich der Mensch eins fühlt mit der Natur. Dieses Naturverhältnis hat seine Wurzeln im mythischen Denken (vgl. Theobald 2003). Es lebt, obwohl weitgehend verdrängt, in uns fort, und in diesem Denken besitzt die Natur einen Eigenwert; denn, mythisch gesehen, wird die Natur als eine Art 'Wesen' aufgefasst, als etwas Personales, weswegen der Mensch sich auch eins fühlen kann mit ihr.

\section{Diskussion}

Es gibt einige Umweltethiker und Umweltethikerinnen, welche die Bedeutung dieser sog. 'Eigenwert-Intuitionen' für die Umweltethik erkannt haben (z.B. Birnbacher 1987, Bosselmann 1989, Krebs 1996 und Gorke 1999). Ohne die Anknüpfung an derartige Emotionen fehle, wie sie zu Recht sehen, der Umweltethik die nötige Motivationskraft (Krebs 1996, 39f.). Aber auch hier kommt wieder die für die Moderne typische Skepsis gegenüber dem Gefühl zum Tragen. Es seien eben nur Emotionen, heißt es, die der Natur einen Eigenwert zubilligten; in Wahrheit sei dieser Eigenwert 'bloßer Schein' (Birnbacher 1980). Diese Einsicht lehre uns die kritische Vernunft, und da wir durch sie wüßten, dass die Natur eigentlich keinen Eigenwert besitze, opferten wir die 'Transparenz unserer Person' (Krebs 1996, 40), wenn wir aus Motivationsgründen an einen Eigenwert der Natur glaubten. Mit solchen Überlegungen wird die Ineffizienz der Umweltethik metaethisch zementiert. Es gibt aber zwei Argumente, die man ihnen entgegenhalten kann und die aus dem scheinbar unauflöslichen Dilemma, in das die Umweltethik sich so begibt, herausführen können. Das eine ist ein philosophisch-metatheoretisches. Es erwächst aus der wissenschaftstheoretischen Selbstreflexion der Vernunft und zeigt, dass sie ohne die dafür notwendige theoretische Legitimation sog. 'nicht-diskursive' Vernunftformen, zu denen das mythische Denken zählt, abwertet, indem sie sich zu Unrecht verabsolutiert (Hübner 1988, Theobald 2003, 135ff.). Dieses Argument steht gewissermaßen in der Tradition der Kritik an der 'Dialektik der Aufklärung'. Das andere Argument ist ein 
psychologisches, das mit jenem konvergiert. Martin Buber hat es in seiner Auseinandersetzung mit der Tiefenpsychologie C.G.Jungs formuliert: "Die Vernunft", sagt Buber, "steckt uns viel zu enge Grenzen und fordert uns auf, nur das Bekannte und auch dies mit Einschränkungen - in bekanntem Rahmen zu leben, so als ob man die wirkliche Ausdehnung des Lebens kennte! Tatsächlich leben wir Tag für Tag weit über die Grenzen unseres Bewusstseins hinaus; ohne unser Wissen lebt das Unbewusste mit. Je mehr die kritische Vernunft vorwaltet, desto ärmer wird das Leben; aber je mehr Unbewusstes, je mehr Mythos wir bewusst zu machen vermögen, desto mehr Leben integrieren wir. Die überschätzte Vernunft hat das mit dem absoluten Staat gemein: unter ihrer Herrschaft verelendet der Einzelne" (Buber 1953, 307). Unter ihrer Herrschaft verelendet auch die Natur, ließe sich mühelos ergänzen. Denn darin sind sich die meisten Umweltethiker einig: dass gerade die Moderne und ihr Vernunftbegriff der Natur- und Umweltzerstörung den Boden bereitet hat - zumindest geistig bzw. 'metaphysisch', wie es heißt (Bayertz 1995, 58).

\section{Schlussfolgerungen}

Will man die oft beklagte Diskrepanz von Einstellung und Verhalten im Bereich des Umweltschutzes überwinden, dann ist es, sofern man dabei auf Ethik setzt, notwendig, das gängige Verständnis von Ethik selbst zu überwinden. In der Moderne als reine 'Reflexionstheorie der Moral' konzipiert, der es hauptsächlich um die Begründung moralischer Normen geht und die ihre Überzeugungskraft im wesentlichen aus der logischen Stringenz von Argumenten bezieht, erweist sich die Ethik als praktisch weitgehend ineffizient. Denn selbst wenn alle Begründungsprobleme gelöst wären, blieben genügend Motivationsprobleme übrig (Ott 2001, 35). Die Umweltethik in ihren Anfängen sah dieses Problem noch in aller Schärfe und forderte, dass es auch eine Aufgabe umweltethischer Reflexion sein müsse zu untersuchen, wie die von ihr entwickelten normativen Orientierungen über ibre theoretische Geltung hinaus zu praktischer Wirksamkeit gebracht werden können (Bayertz 1988, 7). Diese (frühe) programmatische Zielsetzung wurde jedoch nicht weiterverfolgt. Was der politische Philosoph Werner Becker als ein Grunddefizit philosophisch-politischer Reflexion anmahnt, trifft auch auf die philosophischethische Reflexion zu, die ja, wenn es um Fragen der Praxis geht, mit jener konvergiert: "Wissenschaftler und Philosophen neigen dazu, die 'Vernunft' als das ausschlaggebende Rechtfertigungsmittel zur Durchsetzung politischer (bzw. moralischer, ergänzt v. Verf.) Werte und Ziele zu überschätzen. Sie erliegen darin ihrer professionellen Beschränktheit; sie glauben, der Stellenwert, den 'vernünftige' bzw. 'wissenschaftlich-methodische' Argumentationen in ihren Fächern besitzt, müsse in der politischen Dimension der gleiche sein. Das ist ein fundamentaler Irrtum (...). Es nützt nichts, immer wieder von neuem und in modisch wechselnden Gewändern die altehrwürdige Hoffnung der Aufklärung auf das selbständig denkende, allein rationalen Maßstäben vertrauende 'mündige' Individuum zu beschwören (...). Man muss vielmehr die sozialpsychologische Erkenntnis akzeptieren, daß unter den Mitteln zur Erzeugung und Stabilisierung normativer Bindungen die Vernunft - im Unterschied etwa zur Erzeugung emotionaler Bindungen - das schwächste ist" (Becker 1985, 81).

\section{Ausblick}

Wie die gegenwärtige Diskussion über andere aktuelle, gesellschaftlich relevante Themen zeigt, erweist sich das rationalistische Selbstverständnis der Moderne als zunehmend 'antiquiert' (Leicht 2004). Kühne Zeitgeist-Journalisten attestieren ihm sogar eine 'Form der geistigen Magersucht' zu sein (ebd.). Auch wenn solche Bewertungen als überzogen gelten müssen, zeigen sie doch etwas Wichtiges und Richtiges an: das Unbehagen an einer rein wissenschaftlich-rationalen Weltbetrachtung und Daseinsbewältigung. Selbst rationalistische Denker wie Jürgen Habermas suchen neuerdings nach transrationalen Elementen der Verbindlichkeit von Moral und entdecken sie in ursprünglich mythisch-religiösen Gehalten. Man darf hoffen, dass die Signalwirkung, die von derartigen Entwicklungen ausgeht, auch die umweltethische Diskussion affiziert. Erste Ergebnisse in dieser Richtung sind bereits zu beobachten (vgl. Theobald 2003).

\section{Literatur}

Aristoteles: Nikomachische Ethik. Übersetzt und kommentiert von F Dirlmeier. Darmstadt 1979

Augustinus (1925): Über die christliche Lehre. München

Bayertz K (1988): Ökologie und Ethik. Reinbek bei Hamburg

Bayertz K (1995): Eine kurze Geschichte der Herkunft der Verantwortung. In: Ders (Hrsg): Verantwortung. Prinzip oder Problem? Darmstadt

Becker W (1985): Elemente der Demokratie. Stuttgart

Bickman L (1972): Environmental Attitudes and Actions. Journal of Social Psychology 87, 323-324

Birnbacher D (1980): Sind wir für die Natur verantwortlich? In: Ders (Hrsg): Ökologie und Ethik. Stuttgart

Birnbacher D (1987): Ethical principles versus guiding principles in environmental ethics. Philosophica 39 (1) 59-76

Birnbacher D (1998): Utilitaristische Umweltbewertung. In: Theobald W (Hrsg): Integrative Umweltbewertung. Theorie und Beispiele aus der Praxis. Berlin, Heidelberg, New York

Bosselmann K (1989): Im Namen der Natur. Bern

Buber M (1953): Gottesfinsternis. Betrachtungen zur Beziehung zwischen Religion und Philosophie. Zürich

Gorke M (1999): Artensterben. Von der ökologischen Theorie zum Eigenwert der Natur. Stuttgart

Haan G de, Kuckartz U (1996): Umweltbewusstsein. Denken und Handeln in Umweltkrisen. Opladen

Hübner K (1988): Reflexion und Selbstreflexion der Metaphysik. In: Sitzungsberichte der Sudetendeutschen Akademie der Wissenschaften und Künste 1988 (6) 193-202

Krebs A (1996): Philosophische Überlegungen zum Eigenwert der Natur. In: Nutzinger HG (Hrsg): Naturschutz - Ethik - Ökonomie: Theoretische Begründungen und praktische Konsequenzen. Marburg

Krebs A (1997): Naturethik im Überblick. In: Dies (Hrsg): Naturethik. Grundtexte der gegenwärtigen tier- und ökoethischen Diskussion. Frankfurt am Main

Leicht R (2004): Störfaktor Religion. Die Zeit 16, 1

Ott K (2001): Moralbegründungen. Hamburg

Ott K (2002): Ethische Aspekte des Klimawandels. In: Schröder M et al. (Hrsg): Klimavorhersage und Klimavorsorge. Berlin, Heidelberg

SRU (Der Rat von Sachverständigen für Umweltfragen) (2002): Umweltgutachten 2002. Stuttgart

Theobald W (1998): Umwelt und Ethik. Sinn und Unsinn bereichsspezifischer Ethiken für eine integrative Umweltbewertung. In: Schröder W, Daschkeit A (Hrsg): Umweltforschung quergedacht. Perspektiven integrativer Umweltforschung und -lehre. Berlin, Heidelberg, New York

Theobald W (2003): Mythos Natur. Die geistigen Grundlagen der Umweltbewegung. Darmstadt

Eingegangen: 20. Januar 2004 Akzeptiert: 06. Juni 2004 OnlineFirst: 07. Juni 2004 